\title{
MAXIMUM AND MINIMUM PRINCIPLES FOR CAPILLARY SURFACE PROBLEMS WITH PRESCRIBED CONTACT ANGLE
}

\author{
N. ANDERSON and A. M. ARTHURS
}

(Received 22 October 1977)

(Revised 5 March 1978)

\begin{abstract}
Maximum and minimum principles for capillary surface problems with prescribed contact angle are derived in a unified manner from canonical variational theory. The results are illustrated by calculations for a liquid in a cylindrical container with circular cross-section.
\end{abstract}

\section{Introduction}

The nonlinear boundary value problem described by the equations

with

$$
\operatorname{div}\{W(\phi) \operatorname{grad} \phi\}=\phi \text { in } V,
$$

n. $W(\phi) \operatorname{grad} \phi=\cos \gamma$ on $B$,

where

$$
W(\phi)=\left(1+|\operatorname{grad} \phi|^{2}\right)^{-1}
$$

arises in the determination of an equilibrium-free surface $S$ of a liquid that partially fills a cylindrical container under surface forces; gravitational forces and boundary adhesion (cf. Adams [1]). Here $\phi=\phi(x, y)$ represents the height of the capillary surface $S, \gamma$ is the angle of intersection of $S$ and the cylindrical container (measured interior to the liquid) and $\mathbf{n}$ is the outward unit normal field on the boundary $B$ of the cross-section $V$ of the cylinder. For boundaries $B$ of class $C^{4}$ and $\gamma>0$, it has been shown (Spruck [3]) that a solution of the problem exists and is unique. We shall suppose that these conditions are satisfied, and turn to the question of effective methods of solving such problems. 
Because of its nonlinear form, the boundary value problem described by equations (1.1)-(1.3) cannot be solved exactly. Approximate methods are therefore required and in this paper we develop extremum principles which can be used as a basis for variational methods of solution. Our approach employs the canonical theory of complementary variational principles which leads to maximum and minimum principles in a unified manner.

\section{Hamiltonian formalism}

Since we want to use a canonical approach, we set

$$
W(\Phi) \operatorname{grad} \Phi=\mathbf{U},
$$

and then equations (1.1)-(1.3) may be written in Hamiltonian form

$$
\begin{aligned}
\operatorname{grad} \Phi & =\mathbf{U}(1-\mathbf{U} \cdot \mathbf{U})^{-\frac{1}{2}}=\frac{\partial H}{\partial \mathbf{U}} \text { in } V, \\
-\operatorname{div} \mathbf{U} & =-\Phi=\frac{\partial H}{\partial \Phi} \text { in } V, \\
\mathbf{n} \cdot \mathbf{U} & =\cos \gamma \quad \text { on } B .
\end{aligned}
$$

A suitable Hamiltonian $H$ in equations (2.2) and (2.3) is given by

$$
H(\mathrm{U}, \Phi)=-(1-\mathrm{U} \cdot \mathrm{U})^{\frac{1}{2}}-\frac{1}{2} \Phi^{2} .
$$

The exact solution of this problem in (2.2)-(2.4) will be denoted by (u, $\phi)$. From the form of these canonical equations we see that we are dealing with an example of a quasilinear Neumann-type probem.

\section{Variational principles}

The Hamiltonian equations (2.2)-(2.4) can be given a variational description if we introduce the associated canonical action integral (cf. Arthurs [2]):

$$
\begin{aligned}
I(\mathbf{U} \Phi) & =\int_{V}\{\mathbf{U} \cdot \operatorname{grad} \Phi-H(\mathbf{U}, \Phi)\} d x d y-\int_{B} \Phi \cos \gamma d s \\
& =\int_{V}\{(-\operatorname{div} \mathbf{U}) \Phi-H(\mathbf{U}, \Phi)\} d x d y+\int_{B} \Phi(\mathbf{U}-\mathbf{n} \cos \gamma) \cdot \mathbf{n} d s .
\end{aligned}
$$


This is defined for spaces $\Omega_{u}$ and $\Omega_{\phi}$ of continuously differentiable squareintegrable functions $\mathbf{U}$ and $\Phi$ and to keep $H$ real we impose the further condition on $\mathbf{U}$

$$
\text { U. } \mathbf{U}<1 \text {, }
$$

which is satisfied by the exact function $\mathbf{u}$ (see equation (2.1)). This action is stationary at the exact solution ( $u, \phi)$ of equations (2.2)-(2.5).

To go further and obtain extremum principles we define two subsets of $\Omega=\Omega_{u} \times \Omega_{\phi}$ by

$$
\Omega_{1}=\left\{\left(\mathbf{u}_{1}, \phi_{1}\right): \mathbf{u}_{1}=W\left(\phi_{1}\right) \operatorname{grad} \phi_{1}\right\}
$$

and

$$
\Omega_{2}=\left\{\left(\mathbf{u}_{2}, \phi_{2}\right): \operatorname{div} \mathbf{u}_{2}=\phi_{2} \text { in } V, \mathbf{n} \cdot \mathbf{u}_{2}=\cos \gamma \text { on } B\right\} .
$$

These subsets intersect at the exact solution ( $u, \phi)$ of the problem in (2.2) to (2.5).

Using the action and these two subsets we can define functionals $J$ and $G$ by setting

$$
J\left(\phi_{1}\right)=I\left(\mathbf{u}_{1}, \phi_{1}\right) \text { via (3.1), with }\left(\mathbf{u}_{1}, \phi_{1}\right) \text { in } \Omega_{1},
$$

and

$$
G\left(u_{2}\right)=I\left(u_{2}, \phi_{2}\right) \quad \text { via }(3.2) \text {, with }\left(u_{2}, \phi_{2}\right) \text { in } \Omega_{2} .
$$

These lead to the expressions

$$
J\left(\phi_{1}\right)=\int_{V}\left\{\left(1+\left|\operatorname{grad} \phi_{1}\right|^{2}\right)^{\frac{1}{2}}+\frac{1}{2} \phi_{1}^{2}\right\} d x d y-\int_{B} \phi_{1} \cos \gamma d s
$$

and

$$
G\left(\mathbf{u}_{2}\right)=\int_{\nabla}\left\{\left(1-\mathbf{u}_{2} \cdot \mathbf{u}_{2}\right)^{\frac{1}{t}}-\frac{1}{2}\left(\operatorname{div} \mathbf{u}_{2}\right)^{2}\right\} d x d y .
$$

It follows from these definitions that $J\left(\phi_{1}\right)$ is stationary at $\phi$ and $G\left(\mathbf{u}_{2}\right)$ is stationary at $u$. In addition we find that

$$
\begin{aligned}
J\left(\phi_{1}\right)-J(\phi)=\int_{V}\{ & H\left(\mathbf{u}, \phi_{1}\right)-H\left(\mathbf{u}_{1}, \phi_{1}\right)-\left(\mathbf{u}-\mathbf{u}_{1}\right) \cdot \partial H / \partial \mathbf{u}_{1} \\
& \left.-\left[H\left(\mathbf{u}, \phi_{1}\right)-H(\mathbf{u}, \phi)-\left(\phi_{1}-\phi\right) \partial H / \partial \phi\right]\right\} d x d y
\end{aligned}
$$

and

$$
\begin{aligned}
G(\mathbf{u})-G\left(\mathbf{u}_{2}\right)= & \int_{\nabla}\left\{H\left(\mathbf{u}_{2}, \phi\right)-H(\mathbf{u}, \phi)-\left(\mathbf{u}_{2}-\mathbf{u}\right) . \partial H / \partial \mathbf{u}\right. \\
& \left.-\left[H\left(\mathbf{u}_{2}, \phi\right)-H\left(\mathbf{u}_{2}, \phi_{2}\right)-\left(\phi-\phi_{2}\right) \partial H / \partial \phi_{2}\right]\right\} d x d y .
\end{aligned}
$$

Now the Hamiltonian $H(\mathrm{U}, \Phi)$ in (2.5) is convex in $\mathrm{U}$ for all functions $\mathrm{U}$ such that

$$
1-\mathbf{U} . \mathbf{U} \geqslant 0 \text {, }
$$


and strictly concave in $\Phi$ for all functions $\Phi$. Since $u_{1}\left(\phi_{1}\right)=W\left(\phi_{1}\right) \operatorname{grad} \phi_{1}$ automatically satisfies (3.12), the expression (3.10) gives the global minimum principle

$$
J(\phi) \leqslant J\left(\phi_{1}\right) .
$$

Also, if we impose condition (3.12) on $\mathbf{u}_{2}$, that is

$$
\mathbf{u}_{2} \cdot \mathbf{u}_{2} \leqslant 1 \text {, }
$$

expression (3.11) gives the global maximum principle

$$
G\left(\mathbf{u}_{2}\right) \leqslant G(\mathbf{u}) \text {. }
$$

Combining (3.13) and (3.15) we therefore obtain the global complementary variational principles

$$
G\left(\mathbf{u}_{2}\right) \leqslant G(\mathbf{u})=I(\mathbf{u}, \phi)=J(\phi) \leqslant J\left(\phi_{1}\right),
$$

equality holding when $\phi_{1}=\phi$ and $\mathbf{u}_{2}=\mathbf{u}$. The minimum principle for $J$ in (3.16) is just the Euler-Lagrange extremum principle for problems described by (1.1)-(1.3), while the complementary maximum principle for $G$ appears to be new.

\section{Example}

To illustrate these results we take the case of a liquid in a cylindrical container with circular cross-section of radius one. We select two vectors $\left(\mathbf{u}_{1}, \phi_{1}\right)$ and $\left(\mathbf{u}_{2}, \phi_{2}\right)$ in $\Omega_{1}$ and $\Omega_{2}$ respectively:

and

$$
\begin{aligned}
& \phi_{1}=a_{1}+a_{2} r^{2}+a_{3} r^{3}+a_{4} r^{4}, 0 \leqslant r \leqslant 1, \\
& \mathbf{u}_{1}=W\left(\phi_{1}\right) \operatorname{grad} \phi_{1}
\end{aligned}
$$

$$
\begin{aligned}
& \mathbf{u}_{2}=\mathbf{n} \cos \gamma\left\{\alpha r+\sum_{k=1}^{4} b_{k} r^{k+1}\right\}, \quad 0 \leqslant r \leqslant 1, \\
& \phi_{2}=\operatorname{div} \mathbf{u}_{2} \text { in } V,
\end{aligned}
$$

where

$$
\alpha=1-\sum_{k=1}^{4} b_{k} .
$$

In choosing these functions we have imposed extra symmetry properties, namely, $\operatorname{grad} \phi_{1}=0$ and $\mathbf{u}_{2}=0$ at $r=0$, which are satisfied by the exact functions $\phi$ and $\mathbf{u}$. There are eight parameters which are optimised by minimizing $J$ and maximizing $G$. Taking the contact angle $\gamma$ equal to $\pi / 3$ we have carried out the optimization and the resulting parameters are given in Table 1. 
The closeness of the functionals $J$ and $G$ indicates that, in terms of the action metric, the variational solution (4.1) provides an accurate representation of the capillary surface $S$ of the liquid in this case.

TABLE 1

Variational parameters for $\gamma=\pi / 3$

\begin{tabular}{ccccc}
\hline$a_{1}$ & $a_{1}$ & $a_{3}$ & $a_{4}$ & $J$ \\
0.886961 & 0.148485 & 0.108400 & -0.013280 & 1.37425 \\
$b_{1}$ & $b_{2}$ & $b_{3}$ & & \\
0.104994 & -0.038556 & 0.070206 & 0.006850 & 1.37413 \\
\hline
\end{tabular}

\section{References}

[1] N. K. Adams, The physics and chemistry of surfaces (New York, Dover, 1968).

[2] A. M. Arthurs, Complementary variational principles (Oxford, Clarendon Press, 1970).

[3] J. Spruck, Comm. Pure Appl. Math. 28 (1975), 189-200.

Department of Mathematics

University of York

Heslington

York, England YO1 5DD 